\title{
A Novel Fabrication Method for Photonic Lanterns
}

\section{Mariam Mathew, Neethu; Grüner-Nielsen, Lars Erik; Usuga Castaneda, Mario A.; Rottwitt, Karsten}

\section{Published in:}

Optical Fiber Communication Conference 2018

Link to article, DOI:

10.1364/OFC.2018.M4D.4

Publication date:

2018

Document Version

Peer reviewed version

Link back to DTU Orbit

Citation $(A P A)$ :

Mariam Mathew, N., Grüner-Nielsen, L. E., Usuga Castaneda, M. A., \& Rottwitt, K. (2018). A Novel Fabrication Method for Photonic Lanterns. In Optical Fiber Communication Conference 2018 (pp. 1-3). Optical Society of America (OSA). https://doi.org/10.1364/OFC.2018.M4D.4

\section{General rights}

Copyright and moral rights for the publications made accessible in the public portal are retained by the authors and/or other copyright owners and it is a condition of accessing publications that users recognise and abide by the legal requirements associated with these rights.

- Users may download and print one copy of any publication from the public portal for the purpose of private study or research.

- You may not further distribute the material or use it for any profit-making activity or commercial gain

- You may freely distribute the URL identifying the publication in the public portal 


\title{
A Novel Fabrication Method for Photonic Lanterns
}

\author{
Neethu Mariam Mathew ${ }^{1}$, Lars Grüner-Nielsen ${ }^{2}$, Mario A Usuga Castaneda ${ }^{1}$, \\ Karsten Rottwitt ${ }^{1}$ \\ ${ }^{I}$ DTU Fotonik, Ørsteds Plads, building 3432800 Kgs. Lyngby, Denmark. \\ ${ }^{2}$ Danish Optical Fiber Innovation, Åvendingen 22A, 2700 Brønsh $\phi$, Denmark. \\ Corresponding author: karo@fotonik.dtu.dk
}

\begin{abstract}
A new fabrication method for photonic lanterns using a low index UV curable polymer is presented. The insertion loss is predicted, and measurements show a loss lower than $3 \mathrm{~dB}$.
\end{abstract}

OCIS codes: (060.2340) Fiber optics components, (060.4230) Multiplexing.

\section{Introduction}

Single mode fibers (SMFs) will soon be unable to support our continued request for higher data capacity. Space division multiplexing (SDM) has emerged as one solution for this challenge by making use of the spatial modes in a multimode fiber (MMF) [1] or separate cores of multicore fibers [2]. One disadvantage of SDM systems is that each of its components can cause undesirable mode mixing. This can be compensated by using Multiple Input Multiple Output (MIMO) processing, but since MIMO is expensive and adds complexity to the system, it is preferable to avoid it, by engineering better components that preserve the purity of the modes involved. To directly excite the MMF modes, bulk optical set ups using phase masks or spatial light modulators were used earlier. Moreover, due to passive beam combining, coupling loss is proportional to the number of modes in these free space setups. Thus, a low loss mode multiplexer that support a large number of modes is highly on demand. Photonic lanterns are a promising technology to address this need by allowing low loss transmission from a multimode system to independent single mode systems and vice versa [3]. The first lanterns were fabricated using a drawing tower fabrication method [4]. A more applicable fabrication method was first demonstrated by Noordegraaf et al in 2009 [5] where the SMFs are bundled together in a low refractive index fluorine doped silica capillary tube and is fused and tapered together in a glass processor machine [3]. At the taper waist each SMF cores nearly vanish and the SMF cladding becomes the new multimode core and the low index capillary act as the new multi-mode cladding. However fluorine doped silica capillary tubes are expensive and not always available with the desired refractive index. Furthermore, as the number of SMFs increases, the diameter of the capillary should be larger and uniform tapering in a glass processor machine gets difficult.

Here we present a new fabrication method without the need of a low index capillary tube. The fibers are simply crossed so they hold together and are fused and tapered together in a glass processor. The tapered waist is cleaved and spliced to the few mode fiber. As the last step, a UV curable polymer is coated over the tapered part and cured. The total insertion loss measured on a lantern with three similar SMFs when spliced to a two mode fiber is lower than $3 \mathrm{~dB}$.

\section{Fabrication technique}

A photonic lantern is fabricated using three single mode fibers of core diameter $8 \mu \mathrm{m}$ and cladding diameter of 125 $\mu \mathrm{m}$. The coating is removed over a length of $5 \mathrm{~cm}$ and the uncoated parts of the three fibers are crossed together. While crossing the fibers, one end is held free so that the fibers are not twisted but only crossed. The crossed fiber bundle is held between the fiber holding blocks of a Vytran glass processor. The fiber bundle is first fused together using axial heating steps using a FTAT3 graphite filament [6], where the filament runs longitudinally along the fiber bundle axis, back and forth twice. After the axial heating the fused fibers are tapered using a taper length of $20 \mathrm{~mm}$ and a taper ratio (scale factor) of 0.09 . This long taper length of $20 \mathrm{~mm}$ is used to ensure an adiabatic mode transition and thus mode coupling to higher order modes is avoided [3]. If the tapering is done without the axial heating steps, the fibers taper individually as shown in figure 1(a), while the axial heating helps to fuse the fibers well together as in figure 1(b). The tapered fiber bundle is held stretched by a tension of $7 \mathrm{~g}$ between the fiber holding blocks and cleaved using a ruby hand cleaver. The uptaper length, as shown in figure 1(b), is kept short at $1 \mathrm{~mm}$ for better visualization and control of the taper process. The taper width is checked with a view angle of $0^{\circ}$ and $90^{\circ}$ and the cross-section images as in figure 2(a) is taken using end view camera. The cross section images confirm that there is no air gap between the fused fibers 


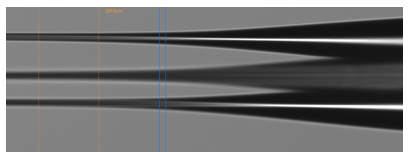

(a)

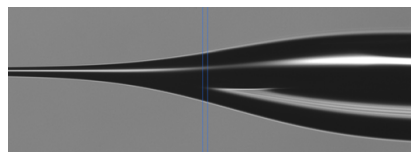

(b)

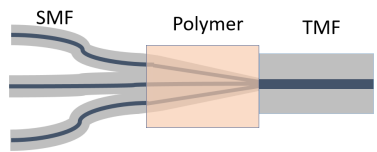

(c)

Fig. 1. Fiber bundle tapering. a) The uptaper where fibers are not fused together. b) The uptaper showing the fibers melt together. c) A schematic representation of the fabricated lantern, TMF: Two mode fiber.
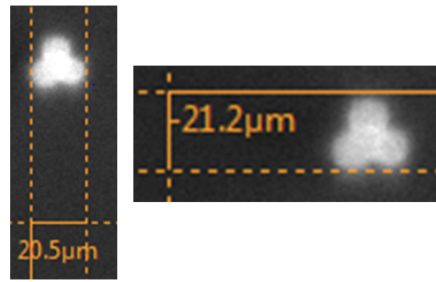

(a)

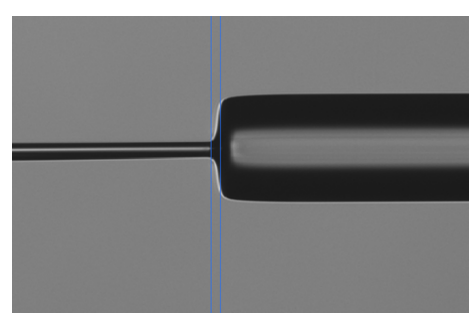

(b)

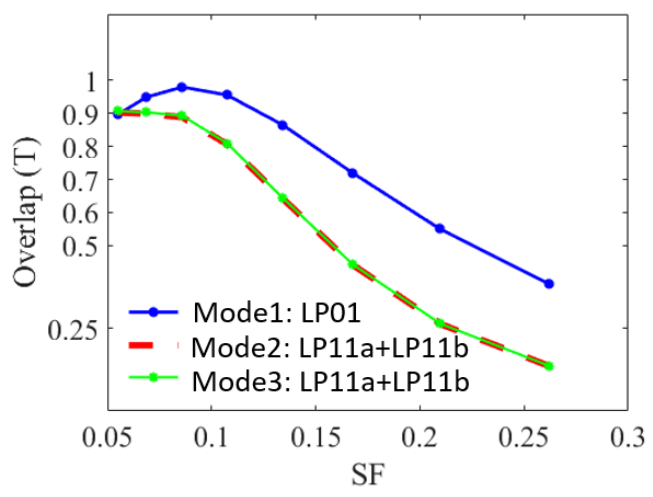

(c)

Fig. 2. Experimental and simulated results on the fabricated lantern. a) Taper cross section for $21 \mu \mathrm{m}$ taper width. b) The dissimilar splice of $21 \mu \mathrm{m}$ tapered tip to a $125 \mu \mathrm{m}$ two mode fiber. c) Simulated mode overlap versus scale factor (SF). $\mathrm{LP}_{11 a}$ and $\mathrm{LP}_{11 b}$ modes have almost the same overlap.

at the multimode end of the taper. The cleaved tapered fiber bundle is spliced to the two-mode step index fiber of core diameter $19.8 \mu \mathrm{m}$ and cladding diameter $125 \mu \mathrm{m}$ as in figure 2(b). A polymer cladding resin (SPC 444 from FOSPIA) having a lower index than the SMF cladding is coated on the spliced lantern, along the taper, covering the splice and UV-cured for 6 minutes. A schematic representation of the fabricated lantern is shown in figure 1(c). The 2D mode profiles at the tapered end are obtained by solving the 2D scalar wave equation. The overlap of the three lantern modes with the $\mathrm{LP}_{01}, \mathrm{LP}_{11 a}$ and $\mathrm{LP}_{11 b}$ modes of the two-mode fiber are calculated as

$$
T=\left|\int_{-\infty}^{\infty} \int_{-\infty}^{\infty} \psi_{1} \psi_{2}^{*} d x d y\right|^{2}
$$

where $\psi_{1}$ and $\psi_{2}$ are the normalized overlaping modes. The calculated overlaps of the lantern modes with the twomode fiber modes varies with scale factor as shown in figure 2(c). The mode overlaps reach the maximum value at a scale factor of 0.09 as in figure 2(c), which corresponds to a taper width of around $21 \mu \mathrm{m}$. While fabricating the lantern, the taper ratio is selected as 0.09 , to utilize this maximum mode overlaps.

\section{Characterization of the lantern}

The insertion loss is measured on six lanterns using a laser source at $1550 \mathrm{~nm}$. The accuracy of the splice alignment of tapered fiber end with the two mode fiber seem to be the most determining factor for lantern loss. A slight splice offset seems to increase the loss considerably. The effect of splice offset on the lantern loss is studied and shown in figure 3. The laser is connected firstly to the two-mode fiber end, through a splice to a SMF to ensure launching of only $\mathrm{LP}_{01}$ mode, and power is measured at each of the three SMFs and the measured total loss is shown by the red marks in figure 3(a). Secondly, the laser is sent through each of the SMFs and the power at the two-mode fiber end is measured each time and showed in figure 3(b). The simulated total loss on all three SMFs, when laser is launched to $\mathrm{LP}_{01}$ of the two-mode fiber end for different splice offsets are shown by continuous lines in figure 3(a). The simulated 


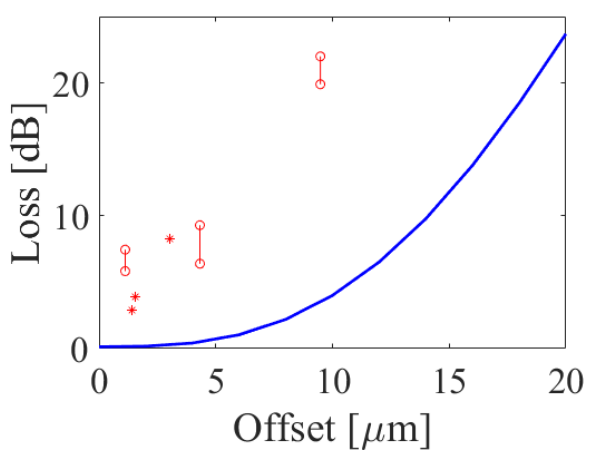

(a)

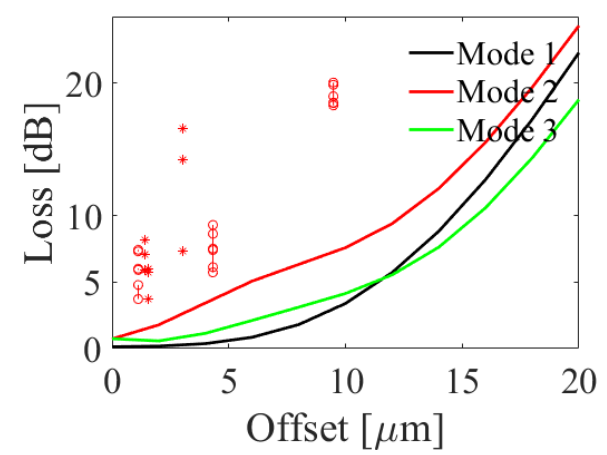

(b)

Fig. 3. Variation of loss with splice offset distance. a) Total measured (marks) and simulated loss (line) out of all the three SMFs with input to the $\mathrm{LP}_{01}$ of two-mode fiber $\mathbf{b}$ ) Loss measured with input to each of the SMF, and Loss simulated for each of the lantern modes. The measured polarization dependent losses for three lanterns is showed by the round marks in both $\mathbf{a}$ and $\mathbf{b}$.

loss of three lantern modes is shown in figure 3(b) as well. The experimental loss seem to be higher than the simulated loss, but follow the same trend for dependence of offset. The variation in the measured loss for a single lantern is due to polarization dependence. The polarization dependent losses are measured on three lanterns as showed by round red marks.

Simulations revealed that for some section of the down taper, the effective indices of the three lantern modes are very close and the modes overlap, which will result in mode coupling. This mode coupling depend on various parameters including the polarization of light. It is observed that the measured loss variations are in same order as the simulated variation in loss between the different lantern modes. The rounding of the two mode fiber during the splice as seen in figure 2(b), can also contribute to loss, which is not considered in the simulations.

\section{Conclusion}

A new fabrication technique for photonic lanterns is presented. A UV curable polymer resin is used as cladding material instead of fluorine doped silica capillary tube. The tapered end of the lantern is spliced to the two-mode fiber before the application of the polymer. Photonic lanterns with three similar SM fibers are fabricated using this new technique. The insertion loss of the lantern is measured and its effect on splice offset is studied. The measured loss seem to depend on the polarization of the input light. Further investigation on the rounding of the spliced two mode fiber could also explain the variation of experimental results from simulations.

Acknowledgements: The Danish Council for Independent research, grant DFF-4184-00433 "Signal processing using higher order modes in optical fibers," is acknowledged for financial support.

\section{References}

1. S. Berdagu et.al., "Mode division multiplexing in optical fibers," Appl.Opt. 21,1950-1955 (1982).

2. S. Matsuo et.al., "12-core fiber with one ring structure for extremely large capacity transmission," Opt. Express 20, 28398-28408 (2012).

3. S.G. Leon-Saval et. al., "Photonic lantern as mode multiplexer for multimode optical communications",Opt.Fiber Technol, 35, 46-55 (2017).

4. S.G. Leon-Saval et.al., " Multimode fiber devices with single-mode performance”, Opt. Lett. 30, 2545-2547 (2005).

5. D. Noordegraaf, "Efficient multi-mode to single-mode coupling in a photonic lantern", Opt. Express 17, 19881994 (2009).

6. US patent no.005935288A, "Method for producing fused fiber bundles", (1997).

7. T.A.Birks et.al.,"'Photonic lantern" spectral filters in multi-core fiber," Opt.Express 20, 13996-14008 (2012) 